\title{
Biomarkers and Bioactivity in Drug Discovery using a Joint Modelling Approach
}

\author{
Kawsher Rahman
}

Lecturer of Anatomy, Faculty of International Study (Medical Sciences), Jiujiang University, Jiujiang, Jiangxi, CHINA

\begin{abstract}
Biomarkers that are validated and robust are required for the enhancement of diagnosis, the observation of drug-related activity, therapeutic reactions, and as the blueprint for developing safer and more direct therapeutic efforts for a variety of chronic ailments. Various kinds of biomarkers have proven impactful when it comes to the discovery and development of drugs, but the procedure that involves identifying and verifying ailment-specific biomarkers has proven to be hassling. In recent times, there have been some advancements in multiple omics (also known as multi-omics) methods like transcriptomic, cytometry, genomics, proteomics, metabolomics and imaging. These advancements have made it possible for the discovery and development of distinct biomarkers for complicated chronic ailments to be accelerated expeditiously. In spite of the fact that numerous drawbacks still need to be looked into, ongoing efforts for the discovery and improvement of illness-associated biomarkers will go a long way in optimizing decision-making across the entire process of drug development and expand our comprehension of the infection processes. In addition, when the preclinical biomarkers are effectively translated into the clinic, the way will pave well to an equally effective implementation of personalized therapies throughout complicated illness environments to become beneficial to patients, healthcare service providers and the industry of bio-pharma.
\end{abstract}

Keywords: Biomarker, Bioactivity, Bio-pharma, Drug Discovery, Joint Modelling Approach

\begin{tabular}{l} 
Manuscript Received: 5 July 2021 \\
\hline $\begin{array}{l}\text { This article is is licensed under a Creative Commons Attribution-NonCommercial 4.0 International License. } \\
\text { Attribution-NonCommercial (CC BY-NC) license lets others remix, tweak, and build upon work non-commercially, and although the new works must also } \\
\text { acknowledge and be non-commercial. }\end{array}$ CC)
\end{tabular}

\section{INTRODUCTION}

Over the years, the movements that have occurred in the field of biomedical research have raised anticipations for more secure and more effective therapies available to those in need of them. Be as it may, the ability of the drug industry to efficiently interpret biomedical research into new market therapies has been hamstrung by critical hurdles (Amin \& Manavalan, 2017; Abedin et al., 2012; Siddique \& Ahmed, 2015; Begum et al., 2012). Discouragingly, there is an increased attrition rate in the clinical attempts that are more than 90 percent (Bai et al., 2013). That means, the success likelihood for drug candidate or biologics entry, the first phase of the trial is estimated at 8 percent (Benjamini and Hochberg, 1995; Manavalan, 2019).

The relatively low success probability from FIH (first-in-human) to NDA (new drug implementation) can be described by one or many purposes, including individual pharmacogenetic heterogeneity to drug response and treatment, the scarcity of biomarkers, lack of security and drug efficacy, and slim therapeutic changes. When the adverse effects are non-evadable, a premature detection at the clinical trial stage is a critical step (Neogy and Ahmed, 2015; Azad et al., 2011; Ahmed et al., 2011; Manavalan, 2020; Donepudi, 2016; Bynagari, 2020). Furthermore, the objective is to design precision drugs and effectively stratify patients according to their ailment type or response medication for personalized therapies. 
To successfully treat complicated chronic ailments (even those in the inflammatory, autoimmune and metabolic diseases and to also develop precision drugs and personalized therapeutic solutions, monitoring the molecular framework of ailment catalyzation is essential prior to, during and right after therapeutic intervention (Buyse and Molenberghs. 1998). As such, a more encompassing comprehension of the elements that factor into drug response has culminated into a rising demand for extra drug efficacy, safety testing and specificity while they are being developed. Something else that has seen increase is pressure from the public for more extensive drug tests for human-friendliness prior to approving them.

\section{Biomarkers in Drug Discovery and DeVELopMent}

Regardless of this new work path to drug development, one which has increased in both cost and time, examining the development of new therapeutic solutions for the eradication of AIDS is an interesting endeavor, especially as a case study for the contrivance of surrogate endpoint biomarkers for separate ailments. It was about 3 years ago that the average AIDS drug was developed, due to the reality that the FDA has given the greenlight to using "viral load" as a replacement biomarker to quantify the probable clinical upside following the verification of the mortality advantage in the fourth-stage research after registration (Donepudi, 2015).

The AIDS drug experience is an acute demonstration of how innovation is capable of accelerating the design of pills without jeopardizing consumer safety. Because of this, clinically relevant and validated surrogate last-mile biomarkers are not just for rudimentary research, but also for clinical applications and patient categorization since they come in handy for the forecasting of phenotypical and molecular alterations as well as for the evaluation drug efficacy and the human-friendliness of therapeutic solutions for all pharmacy genetically heterogeneous patients.

Considering interpersonal pharmaco genetic distinctions in medicine response, searching for substantially sized and capable biomarkers for human ailments like inflammation, cancer and neurodegeneratives is needed urgently. A good number of research teams have been able to show that genetics-associated polymorphisms have an influence on the sensitivity of pill targets (Ahmed, 2009), drug metabolism (Dearden J. C., 2003), drug supply (Eriksson et al., 2009) and the posed risk of adverse reactions (Fadiel and Naftolin, 2003). Both imaging methods and vitro diagnostics have been used in the revelation of further information regarding ailment subsets. Case in point, gene expression is already being used to augment clinical and histologic evaluations of cancerous tumours, which results in the assessment of the recurrence odds and the essence of adjuvant therapy.

Case in point, the focus could be polymorphic, possess variable expression or be subject to particular modifications which impact function or expression or a specific enzyme/protein as is obtainable in cancer (Göhlman and Tallow, 2009; Gorrini et al., 2013). This strategy proved successful when it was used for drugs like imatinib (Gleevec $\left.{ }^{\circledR}\right)$ and trastuzumab (Herceptin ${ }^{\circledR}$ ) for the categorization of patients (Manavalan, 2019) according to their pharmacogenetic polymorphisms.

\section{THE VALidATION OF BiomarkeRs}

In ailments other than cancer, pharmacogenetic heterogeneity is also in existence, paving the way for the forecasting of the risk factor or severemess associated with the diseases in persons. Case in point, there is the heightened risk of ankylosing spondyliti when it comes to the vector of what is referred to as the HLA-B27 allele (Hasum et al. 2008) and the correlation between the common epitope and rheumatoid arthritis (Manavalan \& Donepudi, 2016). As a result, personalized pharmacogenetic data can contribute to the improvement of medicine development via the reduction of variability and the increment in the size of the medication effect by classifying patients according to their type of condition and treatment response.

Though there have been substantial efforts to develop validated biomarkers for the expedition of clinical trials, the reduction of development costs, the development of precision drug, personalized therapeutics and the classification of individuals with disease conditions, the use of psychometric and imaging approaches have not been widely successful. Biomarkers' contribution in terms of clinical diagnosis have also been unimpressive because, in part, there is a shortage of free-moving workflow linking clinical last-mile biomarker founding efforts with grounded validation techniques (Donepudi, 2014b).

In addition, because there is no sufficient comprehension of the tissue and immune-specific modulation of targets in clinical trials and the lack of understanding regarding target moderation in preclinical vivo models has resulted in below-par outcomes in clinical tests. Partly, this is due to the minute volume of patient samples and the culminating limited statistical energy of clinic-based research. In order to surmount these hurdles, some things need to be followed for an automatic workflow process that involves the discovery of biomarker candidature, standardization, assay validation, verification, and the final application as support diagnostic (Donepudi \& Bynagari, 2021). 
After all, when there is better coordination between the different stakeholders in the process of discovering biomarkers is of the essence. It will need a substantial joint effort from the academia, bio-pharma and regulatory bodies.

\section{TECHNOLOGIES IMPLEMENTED IN THE DISCOVERY OF BIOMARKERS}

For biomarkers, information about them is sourced from human genetics, genetic variability, RNA measurements, metabolites, and of course, proteins. Ipso facto, a good number of methods, including metabolomics, proteomics, genomics and imaging approaches are potentially promising for the generation of a new breed of biomarkers which would be capable of mirroring the disease or medical condition at the molecular level (Manavalan, 2019; Ahmed \& Dey, 2009). The quantity of different biomarkers can be ascertained using bio samples such as urine, blood, cerebrospinal fluid, saliva, bodily tissue, bronchoalveolar lavage, and others. This also includes genomic biomarkers like polymorphisms, gene mutation of transcriptomic biomarkers such as microRNA or miRNA, expression profiling, gene, epigenomics, mapping, haplotype/allele, SNP analysis, non-genomics, glycomis, metabolomics, pharmacogenomics, proteomics, and other small molecule samples.

Researchers are now able to carry out transcriptomic and deep sequencing of mRNA or miRNAs, perform a full genome and predict genetic copy numbers (Ahmed \& Dey, 2009). They can also conduct an analysis of mass spectrometry, which is a medium that enables the sensing, discovery and measurement of given compounds, biomolecules and proteins (Lin et al., 2010). The developments on the part of the complicated process, high throughput, microfluidics and microarray technologies (Martin et al., 2002) and worldwide proteomic and genomic methods have created the avenue for one to attribute the planet's disease-regulated changes. In the process, they can recognize a new crop of biomarkers and related pathways.

Case in point, new-age analytical technologies have been the enabling force behind parallel surveys regarding gene expression, genetic polymorphisms and protein expression in test samples on a global scale (Powis and Kirkpatrick, 2007). These movements have culminated in the profiling of cell populations in the immune system with the aid of a variety of approaches like the multiparameter flow cytometry-which provides functional assessment for living cell populaces-the complex dissection of intra-and-extra-cellular moderators, miRNA profile expressions (Prasanth Kumar et al., 2015; Rogers and Hahn, 2010), histone modification (Manojkumar et al., 2021; Shaib et al., 2013), protein analysis, and genomic-wide (or audience-specific) gene expression profiles (Smyth, 2004).

A high-scale analysis of mRNAs and many proteins with cDNA microarrays and complex protein arrays have undergone descriptions in the study of autoimmune health conditions (Smyth, 2005). In a separate research, the protein expression profiling of patients suffering from osteoarthritis were compared with that of the synovial tissue samples of the patients, thus identifying ailment-specific signatures. The novel biomarker methodologies and types are on the fast lane, as more techniques keep emerging while the existing ones continue to evolve. Due to this, it is more critical (in the process) for one to recognize sizable and clinically significantn end point biomarkers in thier numbers using the compendium of multi-omics methods and verification of said biomarkers in clinically important human populaces to find out of the biomarkers' distribution is Gaussian and if there are substantial disparities existing between race, age or sex.

\section{The Role of Biomarkers in Clinical (ANd Pre-Clinical) Research}

Employing biomarkers from the pre-discovery stages to later drug development as well as decision-making is essential when it comes to assessing the activity existing in animal models, human pharmacology, and link animal through observations like proof of mechanism, evaluating the safety levels in animal designs and ascertaining human safety during the early stages of drug development. In addition, every drug development stage comes with its own set of biomarkers, which could or could not be implementable for other stages. In order to possess value, the biomarkers need to be robust, accessible and reproducible. That is, it needs to be not only measurable but present in body fluids.

Also, a biomarker needs to be sensitive and have a particular ability to discern false negatives from true positives (Manavalan, 2018). They must be capable of indicating the presence of the disease and also interpret the response of the ailment to treatment and time. Most importantly, the detection of a biomarker needs to be clinically significant and be a provider of medical benefits for the patient's good, which is improved survival and quality life. In preclinical studies, biomarker examples include the protein expression of cell surfaces, genomic expression profiles, serum-based chemistries, drug PD/PK quantifications, serum transaminases, receptor occupancy by imaging, and drug supply.

For late drug development, the use of biomarkers include the assessment of optimal regimen and dose response for the sought-after pharmacologic impact, the determination of the roles differences play when it comes to metabolism and safety symbols for the ascertainment of dose response for toxicity levels (Raya et al., 2021). Case in point, once a biomarker is validated, it can be used for selecting dosage in the second and third phases of clinical trials relying on the PD/PK relationship of the biomarker and the forecast therapeutic index in order to discern between compound 
candidature and other normal compounds. Biomarkers that quantify PD/PK relationships also have a hand in the generation of insightful feedback to determine if the mechanism can or cannot be translated into clinical testing.

Audience-specific biomarkers also prove valuable when classifying patients according to their type of health conditions or response rate to medication. This was the strategy that proved effective with imatinib and trastuzumab for patient classification according to the pharmacogenetic polymorphisms they possess. In clinical studies, biomarkers have applied for diagnosis as elements that indicate the progression levels of an ailment. It has also been used to forecast and keep a close eye on clinical response to therapeutic solutions such as PET brain image, blood autoantigens, serum chemistries, electrocardiogram, pulmonary function check, bone densitometric quantification and neonatal Apgar score. In late clinical development, the biomarkers used are pain scales, antimicrobials (or culture testing), psychometric testing, electrocardiogram and pulmonary function tests. After all, biomarkers are potentially capable of revealing the predictive details of the future health condition of a patient-compared to diagnostics which simply group patients for a single time point.

\section{State of Things, Drawbacks \& Future POVs}

Regardless of the fact that DNA microarray studies have culminated in salient developments in research into the world's gene expression profiling of auto-immuneness, their utilization is restricted because there are physiologically important splice variants possessed by every cell population (Sharma et al., 2021). The additional challenge resides in the reality that the huge mRNA proportion is not interpreted as proteins, even if it has been activated at the transcription level.

Proteomics is yet to reach its full potential because it is limited by existing technology, the difficulty associated with identifying low abundance symbolizers, the shortage of quality reagents like informatic tools and monoclonal antibodies (Donepudi, 2014a). Proteomic methods of unbiased nature have experienced insignificant success when it comes to the examination of autoimmune ailments. Meanwhile, FACS and array platforms have been limited due to the fact that it is a bottom-up approach with cross-carpeted knowledge about target proteins. Knowing which salient parts will be omitted as a result of the limitations of existing antibodies or arrays is impossible (Zhu et al., 2021).

Newer methods as regards bioinformatics as well as assay and multi-omics integration platforms must be designed for the simultaneous study of different datasets—-such as gene, protein expression, miRNA expression and FACS initiatives. Using complex methods and multi-omics platforms in analyzing systemic autoimmune ailments will not just recognize a wide array of important biomarkers but also uncover the aspects that need further development. Also, there is an increasing consensus that making for accurateness, efficacy and safety is something that needs more than one biomarker. As a result, early recognition of said biomarkers is important for go and no-go decisions as well as at certain critical stages during the development of drugs.

Contextually, using the multi-omics and complex tech method can bring about a substantial contribution to clinical development at the earliest stages. As a result, coming to an understanding of the upsides and limitations of these strategies and tech-including how they can be used for the discovery of particular biomarkers is of the essence for the efficient realization of biomarkers that are fit for purpose. Another challenge in the field is that of robustness and assay reproducibility. By increasing the number and size of sample replicates-and also incorporating a team of biostatisticians and bioinformatic scientists into the clinical research from the study design stage to the analysis stageought to improve the procedure.

The restricted access to ailment-related tissues is yet another drawback that exists in the field. It has amounted to the widespread employment or auxiliary blood for identifying RNA, DNA, protein and other minute molecular biomarkers, especially in immunity-related ailments. Case in point, world genetic expression profiling now plays the role of a surrogate for bio phenotypes in cell populaces inside the immune system (Bynagari \& Amin, 2019). Though global profiling of either protein or gene expression comes instructive, the evaluation of protein expression or the mRNA alone may not be adequate for the recognition of clinically critical surrogate endpoint biomarkers-the one which can boldly mirror the disease phenotype prior to, during and after therapy has intervened (Bynagari, 2018).

\section{OBservations AND Conclusion}

The majority of emerging biomarkers and their associations with diseases is mostly obtained from two or more exhibits at a given point in time. The approaches favored are, in essence, a snapshot of occurrences for the state of the ailment at a specific time (Ahmed et al., 2021; Doewes et al., 2021; Manojkumar et al., 2021; Panchal et al., 2021; Raya et al., 2021; Sharma et al., 2021; Zhu et al., 2021). Whereas, signalling systems possess dynamic in both their use and their framework. Due to the fact that single measurements do not quite capture the entire dimensions of clinical results, a continuous and multi-dimensional approach is required to substitute for the one-dimensional model. 
Poor test design and technical hamstrings have led to limited success for discovering and validating robust biomarkers as replacement endpoints with the potential to be substituted for the clinically critical endpoints. A more improved and coordinated clinical and experimental design is needed, similar to the essence of more standardized multiparametric tech, multi-omics and analysis platforms. Also, clinicians, scientists, the biopharma sector and the regulatory bodies need to collaborate more to accelerate the discovery of qualified and validated biomarkers to a more reasonable degree.

\section{REFERENCES}

Abedin, M. M. M., Ahmed, A. A. A., and Neogy, T. K. (2012). Mechanism of Accountability and Auditing: Public Sector Scenarios of Bangladesh. Journal of Business Studies, 4, 131-148.

Ahmed, A. A. A. \& Dey, M. M. (2009). Timeliness attributes and the extent of accounting disclosure: a study of banking companies in Bangladesh. Osmania Journal of International Business Studies, 4(1).

Ahmed, A. A. A. (2009). The Effect of Timeliness Regulation of Corporate Financial Reporting: Evidence from Banking Sector of Bangladesh. Accounting and Management Information Systems, 8(2), 216 - 235. http:/ / online-cig.ase.ro/jcig/art/8_2_4.pdf

Ahmed, A. A. A., \& Dey, M. M. (2009). Corporate Attribute and the Extent of Disclosure: A Study of Banking Companies in Bangladesh. Proceedings of the 5th International Management Accounting Conference (IMAC), OCT 19-21, 2009, UKM, Kuala Lumpur, MALAYSIA, Pages: 531-553. https://publons.com/publon/11427801/

Ahmed, A. A. A., Hussain, S., Kurniullah, A. Z., Ramirez-Asis, E., Al-Awawdeh, N., Al-Shamayleh, N. J. M., Julca-Guerrero, F. (2021). Protection against Letters of Credit Fraud. Journal of Legal, Ethical and Regulatory Issues, 24(Special Issue 1), 1-11. https://doi.org/10.5281/zenodo.5507840

Ahmed, A. A. A., Khan, W., \& Hossain, M. S. (2011). Reporting Practice of Accounting Disclosure on Changes in Listed Companies of Bangladesh. ASA University Review, 5(1), 83-96. https://www.researchgate.net/publication/336664901

Amin, R., \& Manavalan, M. (2017). Modeling Long Short-Term Memory in Quantum Optical Experiments. International Journal of Reciprocal Symmetry and Physical Sciences, 4, 6-13. Retrieved from https://upright.pub/index.php/ijrsps/article/view/48

Azad, M. R., Khan, W., \& Ahmed, A. A. A. (2011). HR Practices in Banking Sector on Perceived Employee Performance: A Case of Bangladesh. Eastern University Journal, 3(3), 30-39. https://doi.org/10.5281/zenodo.4043334

Bai, J. P. F., A. V. Alekseyenko, A. Statnikov, I.-M. Wang and P. H. Wong (2013). Strategic applications of gene expression: from drug discovery/development to bedside. AAPS J., 15, 427-437.

Begum, R., Ahmed, A. A. A., \& Neogy. T. K. (2012). Management Decisions and Univariate Analysis: Effects on Corporate Governance in Bangladesh. Journal of Business Studies, 3, 87-115.

Benjamini, Y. and Y. Hochberg (1995). Controlling the false discovery rate: a practical and powerful approach to multiple testing. J. R. Stat. Soc. Series B Stat. Methodol. 57, 289-300.

Buyse, M. and G. Molenberghs (1998). The validation of surrogate endpoints in randomized experiments. Biometrics, 54, $186-201$.

Bynagari, N. B. (2018). On the ChEMBL Platform, a Large-scale Evaluation of Machine Learning Algorithms for Drug Target Prediction. Asian Journal of Applied Science and Engineering, 7, 53-64. Retrieved from https://upright.pub/index.php/ajase/article/view/31

Bynagari, N. B. (2020). The Difficulty of Learning Long-Term Dependencies with Gradient Flow in Recurrent Nets. Engineering International, 8(2), 127-138. https://doi.org/10.18034/ei.v8i2.570

Bynagari, N. B., \& Amin, R. (2019). Information Acquisition Driven by Reinforcement in Non-Deterministic Environments. American Journal of Trade and Policy, 6(3), 107-112. https://doi.org/10.18034/ajtp.v6i3.569

Dearden, J. C. (2003). In silico prediction of drug toxicity. J. Comput. Aided Mol. Des., 17, 119-127.

Doewes, R. I.; Ahmed, A. A. A.; Bhagat, A.; Nair, R.; Donepudi, P. K.; Goon, S.; Jain, V.; Gupta, S.; Rathore, N. K.; Jain, N. K. (2021). A regression analysis based system for sentiment analysis and a method thereof. Australian Official Journal of Patents, 35(17), Patent number: 2021101792. https://lnkd.in/gwsbbXa

Donepudi, P. K. (2014a). Technology Growth in Shipping Industry: An Overview. American Journal of Trade and Policy, 1(3), 137-142. https://doi.org/10.18034/ajtp.v1i3.503

Donepudi, P. K. (2014b). Voice Search Technology: An Overview. Engineering International,2(2), 91-102. https://doi.org/10.18034/ei.v2i2.502

Donepudi, P. K. (2015). Crossing Point of Artificial Intelligence in Cybersecurity. American Journal of Trade and Policy, 2(3), 121128. https:// doi.org/10.18034/ajtp.v2i3.493

Donepudi, P. K. (2016). Influence of Cloud Computing in Business: Are They Robust?. Asian Journal of Applied Science and Engineering, 5(3), 193-196. Retrieved from https://journals.abc.us.org/index.php/ajase/article/view/1181

Donepudi, P. K., \& Bynagari, N. B. (2021). Prediction of Transfusion Based on Machine Learning. Int. J. of Aquatic Science, 12(3), 21682180. http://www.journal-aquaticscience.com/article_136518.html 
Eriksson, S., S. E. Prast-Nielsen, E. Flaberg, L. Szekely and E. Arner (2009). High levels of thioredoxin reductase 1 modulate drugspecific cytotoxic efficacy. Free Radic. Biol. Med., 47, 1661-1671.

Fadiel, A. and F. Naftolin (2003). Microarray applications and challenges: a vast array of possibilities. Reprod. Sci., 1, $1111-1121$.

Gorrini, C., I. S. Harris and T. W. Mak (2013). Modulation of oxidative stress as an anticancer strategy. Nat. Rev. Drug Discov., 12, 931-947.

Hasumi, H., M. Baba, S. Hong, Y. Hasumi, Y. Huang, M. Yao, V. Valera, W. Linehan and L. Schmidt (2008). Identification and characterization of a novel folliculin-interacting protein fnip2. Gene, 415, 60-67.

Lin, D., Z. Shkedy, G. Molenberghs, W. Talloen, H. Gohlmann and L. Bijnens (2010). Selection and evaluation of gene-specific biomarkers in pre-clinical and clinical microarray experiments. Online J. Bioinform., 11, 106-127.

Manavalan, M. (2018). Do Internals of Neural Networks Make Sense in the Context of Hydrology?. Asian Journal of Applied Science and Engineering, 7, 75-84. Retrieved from https://upright.pub/index.php/ajase/article/view/41

Manavalan, M. (2019). P-SVM Gene Selection for Automated Microarray Categorization. International Journal of Reciprocal Symmetry and Physical Sciences, 6, 1-7. Retrieved from https://upright.pub/index.php/ijrsps/article/view/43

Manavalan, M. (2019). Using Fuzzy Equivalence Relations to Model Position Specificity in Sequence Kernels. Asian Journal of Applied Science and Engineering, 8, 51-64. Retrieved from https://upright.pub/index.php/ajase/article/view/42

Manavalan, M. (2020). Intersection of Artificial Intelligence, Machine Learning, and Internet of Things - An Economic Overview. Global Disclosure of Economics and Business, 9(2), 119-128. https://doi.org/10.18034/gdeb.v9i2.584

Manavalan, M., \& Donepudi, P. K. (2016). A Sample-based Criterion for Unsupervised Learning of Complex Models beyond Maximum Likelihood and Density Estimation. ABC Journal of Advanced Research, 5(2), 123-130. https://doi.org/10.18034/abcjar.v5i2.581

Manojkumar, P., Suresh, M., Ahmed, A. A. A., Panchal, H., Rajan, C. C. A., Dheepanchakkravarthy, A., Geetha, A., Gunapriya, B., Mann, S., \& Sadasivuni, K. K. (2021). A novel home automation distributed server management system using Internet of Things. International Journal of Ambient Energy, https://doi.org/10.1080/01430750.2021.1953590

Martin, Y. C., J. L. Kofron and L. M. Traphagen (2002). Do structurally similar molecules have similar biological activity? J. Med. Chem., 45, 4350-4358.

Nantasenamat, C., C. Isarankura-Na-Ayudhya, T. Naenna and V. Prachayasittikul (2009). A practical overview of quantitative structure-activity relationship. EXCLI J., 8, 74-78.

Neogy, T. K. and Ahmed, A. A. A. (2015). The Extent of Disclosure of Different Components of Disclosure Index: A Study on Commercial Banks in Bangladesh. Global Disclosure of Economics and Business, 4(2), 100-110. https://doi.org/10.18034/gdeb.v4i2.139

Panchal, H., Sadasivuni, K. K., Ahmed, A. A. A., Hishan, S. S., Doranehgard, M. H., Essa, F. A., Shanmugan, S., \& Khalid, M. (2021). Graphite powder mixed with black paint on the absorber plate of the solar still to enhance yield: An experimental investigation. Desalination, Volume 520. https://doi.org/10.1016/j.desal.2021.115349

Prasanth Kumar, S., Y. T. Jasrai, H. A. Pandya and R. M. Rawal (2015). Pharmacophore-similarity-based QSAR (PS-QSAR) for groupspecific biological activity predictions. J. Biomol. Struct. Dyn., 33, 56-69.

Raya, I., Kzar, H. H., Mahmoud, Z. H., Ahmed, A. A. A., Ibatova, A. Z., \& Kianfar, E. (2021). A review of gas sensors based on carbon nanomaterial. Carbon Letters. Article No: 276. https://doi.org/10.1007/s42823-021-00276-9

Rogers, D. and Hahn, M. (2010). Extended-connectivity fingerprints. J. Chem. Inf. Model., 50, 742-754.

Shaib, W., R. Mahajan and B. El-Rayes (2013). Markers of resistance to anti-egfr therapy in colorectal cancer. J. Gastrointest. Oncol., $4,303-318$

Sharma, D. K., Chakravarthi, D. S., Shaikh, A. A., Ahmed, A. A. A., Jaiswal, S., Naved, M. (2021). The aspect of vast data management problem in healthcare sector and implementation of cloud computing technique. Materials Today: Proceedings. https://doi.org/10.1016/j.matpr.2021.07.388

Siddique, M. N. \& Ahmed, A. A. A. (2015). Congruence of Competitive Advantage and Transfer Pricing: A Study on Selected MNCs Operating in Bangladesh. Asian Accounting $\mathcal{E}$ Auditing Advancement, 5(2), 119-126. https:/ / www.researchgate.net/publication/354712086

Smyth, G. K. (2004). Linear models and empirical Bayes methods for assessing differential expression in microarray experiments. Stat. Appl. Genet. Mol. Biol., 3, 397-420.

Zhu, Y., Kamal, E. M., Gao, G., Ahmed, A. A. A., Asadullah, A., Donepudi, P. K. (2021). Excellence of Financial Reporting Information and Investment Productivity. International Journal of Nonlinear Analysis and Applications, 12(1), 75-86. https://doi.org/10.22075/ijnaa.2021.4659

$--0--$ 